\title{
Benign Palate Neoplasm
}

National Cancer Institute

\section{Source}

National Cancer Institute. Benign Palate Neoplasm. NCI Thesaurus. Code C4599.

A non-metastasizing neoplasm that arises from the hard palate, soft palate, or uvula. 